\title{
Diagnostik und Therapie bei fehlverheilten Beckenringverletzungen
}

\author{
M. H. Hessmann, P. M. Rommens
}

\section{Zusammenfassung}

Korrektureingriffe bei fehlverheilten Beckenringverletzungen sind komplexe und anspruchsvolle Eingriffe. Der Begriff fehlverheilt beinhaltet drei verschiedene Heilungsprobleme: nicht verheilte Frakturen und/oder Luxationen, unvollständig verheilte Verletzungen und in Fehlstellung verheilte Läsionen. Auch Kombinationen verschiedener Fehlheilungen können vorkommen. Heilungsprobleme können sich sowohl auf den vorderen als auch auf den hinteren Beckenring beziehen. Korrektureingriffe haben nur dann Aussichten auf klinischen Erfolg wenn die vorhandene Beschwerdesymptomatik auf die bestehende Fehlheilung zurückzuführen ist. Eine differenzierte präoperative klinische und radiologische Abklärung ist somit unerlässlich. Außerdem ist eine detaillierte Planung des Operationsablaufs für dessen Gelingen unabdingbare
Voraussetzung. Risiken und zu erwartender Behandlungserfolg müssen vorab mit dem Patienten ausführlich besprochen werden. Korrekturmaßnahmen bei Pseudarthrosen beinhalten die Ausräumung der Instabilitätszone, dessen Auffüllung mit autologer Spongiosa und die stabile Osteosynthese sowohl ventral als auch dorsal. Ausgeheilte Fehlstellungen erfordern eine vorsichtige aber oftmals aufwendige Mobilisation der betroffenen Beckenhälfte mit dreidimensionaler Stellungskorrektur. Mögliche peri- und postoperative Komplikationen sind Schäden von neurovaskulären Strukturen, postoperative Wundheilungsstörungen und Infektionen und Implantatversagen mit Pseudoarthrosenrezidiv. Umfassende Erfahrungen bei der chirurgischen Behandlung akuter Beckenring- und Acetabulumfrakturen sind für das Gelingen von Korrekturmaßnahmen am Becken unabdingbare Voraussetzung.

\section{Einleitung}

Ein besseres Verständnis von Pathophysiologie und Biomechanik instabiler Beckenringfrakturen hat die Versorgungsstrategie dieser Verletzungen in den letzten Jahrzehnten erheblich beeinflusst und optimiert. Durch eine standardisierte Behandlungstaktik, angepasst an das individuelle Verletzungsmuster des einzelnen Patienten, konnten sowohl Morbidität als auch Mortalität bei instabilen Beckenringverletzungen signifikant gesenkt werden [14,15,16]. Während hämodynamische Überlegungen in der Initialphase eine überragende Rolle spielen, ist es das

OP-JOURNAL 2000; 16: 44-50

(c) Georg Thieme Verlag Stuttgart · New York weitere Bestreben des behandelnden Unfallchirurgen - unter Berücksichtigung des jeweiligen lokalen Weichteilschadens, des individuellen osteoligamentären Verletzungsmusters sowie des Allgemeinzustandes des Patienten - die normale Anatomie des Beckenrings wiederherzustellen und die einzelnen osteoligamentären Verletzungen zu einer stabilen Ausheilung zu bringen mit dem Ziel, eine schmerzfreie Vollbelastbarkeit zu ermöglichen [6].

Trotz verbesserter Erkenntnisse und Erfahrungen in der Behandlung instabiler Beckenringverletzungen werden wir aber auch heute noch regelmäßig mit der komplexen Problematik instabil oder fehlverheilter Frakturen konfrontiert. Korrektureingriffe bei Fehlheilun- gen des Beckens sind eine große Herausforderung für den behandelnden Chirurgen und stellen sehr hohe operationstechnische Ansprüche. Ausgedehnte Erfahrungen in der Behandlung frischer Verletzungen sind für das Gelingen unabdingbare Voraussetzung. Als rekonstruktive Eingriffe werden Korrekturen mehr als drei bis vier Monate nach dem Unfallereignis bezeichnet [3].

\section{Behandlungsstrategie bei frischen Beckenringverletzungen}

Bei Patienten mit einer Beckenringinstabilität soll die Behandlung von begleitenden, sofort lebensbedrohlichen Verletzungen das primäre Bestreben des behandelnden Unfallchirurgen sein [14]. Da viele Patienten mit Beckenringfraktur eine hämodynamische Instabilität bis hin zum massiven hämorrhagischen Schock aufweisen, ist die kreislaufmäßige Stabilisierung als akut lebenserhaltende Maßnahme gegenüber der osteoligamentären Rekonstruktion des Beckenrings zunächst vorrangig. Die provisorische Fixierung des Beckenrings trägt durch Stabilisierung der Fraktur, Kompression der Frakturflächen und Reduzierung des intrapelvinen Volumens in entscheidender Weise zur Schockbekämpfung bei. Die notfallmäßige Stabilisierung des Beckenrings kann entweder durch Anlage eines Fixateur externe mit Verankerung der Schanz-Schrauben im supra-acetabulären Knochen oder in der Beckenschaufel oder mit Hilfe einer Beckenzwinge erfolgen.

Während die Beckenzwinge für die definitive Frakturbehandlung nicht geeignet ist, können manche rotationsinstabile Verletzungen mit einem Fixateur externe gut zur Ausheilung gebracht werden. Andere Typ B-Verletzungen, bei denen der ventrale Beckenring mit dem Fixateur nicht ausreichend reponiert, bzw. geschlossen werden kann, bedürfen einer internen Osteosynthese. Ist bei dem Patienten mit einer rotationsinstabilen 
Beckenringfraktur eine Laparotomie erforderlich oder muss eine Blasenverletzung übernäht werden, ist es erstrebenswert den vorderen Beckenring auf dem Rückzug nach der Laparotomie definitiv intern durch Platten- und/oder Schraubenosteosynthese $\mathrm{zu}$ fixieren.

Kombinierte rotatorische und vertikale Instabilitäten erfordern immer eine Stabilisierung des vorderen und des hinteren Beckenrings. Bei den C-Verletzungen ist eine alleinige vordere innere oder äußere Fixation ohne Stabilisierung des hinteren Beckenringes als suboptimale Therapie anzusehen.

Der dorsale Beckenring kann mit einem Fixateur externe allein nicht ausreichend stabilisiert werden [1]. Die Osteosynthese am dorsalen Beckenring kann perkutan oder offen erfolgen, wobei sich - in Abhängigkeit des Frakturtyps - die ventrale Plattenosteosynthese, die sakroiliakale Verschraubung und die Kleinfragmentosteosynthese als Alternativen anbieten $[6,9,16]$.

\section{Diagnostik fehlverheilter Beckenringverletzungen}

Der Begriff fehlverheilt beinhaltet drei verschiedene Heilungsprobleme: nicht verheilte Frakturen und/oder Luxationen, unvollständig verheilte Verletzungen und in Fehlstellung verheilte Läsionen. Auch Kombinationen verschiedener Fehlheilungen können vorkommen. Diese Heilungsprobleme können sich sowohl auf den vorderen als auch auf den hinteren Beckenring beziehen.

\section{Klinische Symptomatik}

Die Mehrzahl der Patienten mit Fehlheilungen im Bereich des Beckens weisen eine komplexe, häufig zunächst nicht exakt zuzuordnende Beschwerdesymptomatik auf. Das klinische Bild einer persistierenden Instabilität wird oftmals durch eine belastungs- und positionsabhängige Schmerzsymptomatik geprägt. Diese Schmerzen können im vorderen oder im hinteren Beckenring lokalisiert sein. Bevor nun aber über Therapieansätze entschieden werden kann, muss der Ursprung des Schmerzes sorgfältig untersucht werden und müssen instabilitätsbedingte Beschwerden von neurologischen, lumbosakralen, sakroiliakal arthrotiformen oder atypischen Schmerzen im Beckenboden differenziert werden. Beckenasymmetrien mit verbliebener vertikalen Dislokation oder Fehlrotation einer Beckenhälfte führen oftmals $\mathrm{zu}$ einer schiefen Sitzposition, einem Beckenschiefstand mit Beinlängendifferenz und Krümmung der Lumbalwirbelsäule (Skoliose) sowie Abweichungen im Gangmuster. Durch vertikale Verschiebung einer Beckenhälfte werden Sakrum und Coccyx mehr prominent, was zu einer entsprechenden Beschwerdesymptomatik beim Sitzen und Liegen führt. Beckenfehlstellungen können sich zudem kosmetisch störend auswirken (siehe auch Abb. 3a), zur Dyspareunie führen, ein Geburtshindernis darstellen oder durch Druck einzelner Knochenfragmente auf Organe eine entsprechend urologische oder intestinale Symptomatik hervorrufen. Andere Patienten weisen neurologische Ausfälle an einer oder beiden unteren Extremitäten auf.

\section{Apparative Diagnostik}

Neben einer ausführlichen Anamnese und einer auf Stabilität und Symmetrie des Beckenrings gerichteten klinischen Untersuchung, sind Röntgenuntersuchungen für die exakte Diagnostik und weitere Therapieplanung unerlässlich [7]. Die Beckenübersichtsaufnahme erlaubt eine Beurteilung der Becken(a)symmetrie. Der Vergleich der Position der beiden Acetabuli vermittelt Informationen über Art und Ausmaß der vorhandenen Rotationsfehlstellung und über die vertikale Dislokation der betroffenen Beckenhälfte. Manchmal sind belastete Aufnahmen im wechselseitigen Einbeinstand erforderlich um eine Symphyseninstabilität nachzuweisen. Die Dorsalverschiebung einer Beckenhälfte lässt sich am besten in der Beckeneingangsaufnahme beurteilen, während der Vertikalversatz am besten anhand der Beckenausgangsaufnahme eingeschätzt werden kann. Eine genaue Analyse dieser drei konventionellen Aufnahmen ermöglicht oft Rückschlüsse über die primäre Läsion. Idealerweise sollten auch die primären Unfallaufnahmen zur Beurteilung vorliegen.

Die Computertomographie ermöglicht eine detaillierte Abklärung der einzelnen Läsionen, insbesondere im dorsalen Beckenringbereich. Um eine räumliche Vorstellung der Beckendeformität zu bekommen, ist die 3D-Rekonstruktion besonders hilfreich. Mit moderner Software kann der spätere Korrektureingriff bereits virtuell am 3D-Modell simuliert werden.

Nachdem nun die Fehlstellung oder Instabilität des Beckenrings als Ursache für die geklagten Beschwerden identifi- ziert worden ist und abgeklärt wurde ob durch einen Korrektureingriff - auch unter Berücksichtigung des operativen Risikos - eine Befundbesserung erreicht werden kann, muss eine genaue chirurgische Behandlungsstrategie ausgearbeitet werden. Die geplante Vorgehensweise sollte ausführlich mit dem Patienten besprochen werden.

Vielmehr noch als bei der Osteosynthese von akuten Beckenringverletzungen ist die Korrektur eines fehlverheilten Beckens ein risikoreiches Unternehmen [8]. Verletzungen neurovaskulärer Strukturen, Wundheilungsstörungen und Infektionen müssen als mögliche Komplikation angesprochen werden. Es sollte auch dem Patienten bewusst sein, dass die knöcherne Heilung nach Korrektur von Pseudarthrosen und etablierte Fehlstellungen länger dauert als bei einer frischen Läsion $[4,8]$.

Mögliche daraus resultierende Probleme sind Implantatlockerungen, Implantatversagen und/oder Auftreten eines Pseudarthrosenrezidivs. Zudem sollte dem Chirurgen und dem Patienten klar sein, dass Art und Dauer der Nachbehandlung nicht derjenigen einer Erstversorgung entspricht. In der Regel beträgt die postoperative Entlastungsdauer nach Beckenkorrektur mindestens 12 Wochen.

\section{Korrekturen bei Pseudarthrosen}

Beckenringinstabilitäten können ventral, dorsal oder kombiniert ventral/dorsal vorkommen. Eine Instabilität im vorderen Beckenring kann in Höhe der Symphyse (Abb.1) oder der Schambeinäste lokalisiert sein. Als operative Zugangswege stehen uns die aus der akuten Chirurgie bekannten konventionellen Zugänge zur Verfügung. Die Symphyse kann in der Regel über einen Pfannenstielschnitt gut erreicht werden. Transpubische Instabilitäten werden durch die medialen Fenster des ilioinguinalen Zugangs angegangen.

Ist die Symphyse instabil, muss das meist sehr derbe Narbengewebe zwischen den beiden Beckenhälften ausgeräumt werden. Um bei dieser Maßnahme Urethraverletzungen zu vermeiden ist es sinnvoll, präoperativ einen transurethralen Blasenkatheter einzubringen, um intraoperativ die Harnröhre besser lokalisieren zu können. Anschließend wird der vordere Beckenring mit einer Zirkelklemme oder Faraboeufzange verschlossen und mit einer Plattenosteosynthese stabilisiert. Wirken erhebliche Rotations- 


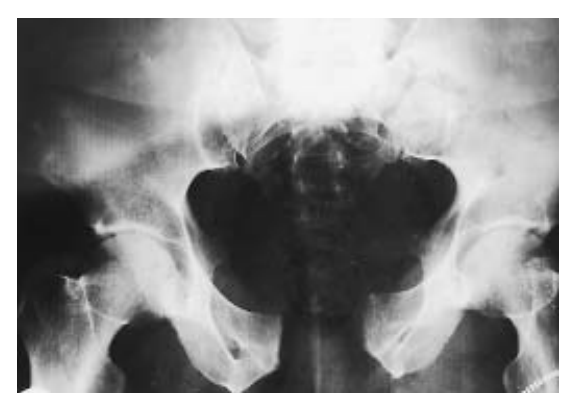

Abb.1 a 55-jähriger Patient mit offener Bekkenringverletzung nach Verkehrsunfall.

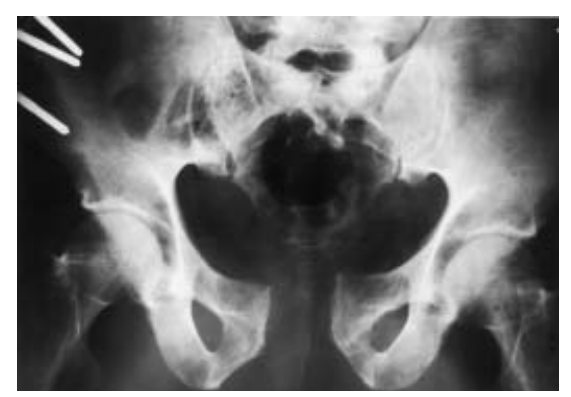

Abb.1 b Primäre Stabilisierung mittels Fixateur externe. Persistierende Diastase der Symphysis pubis.

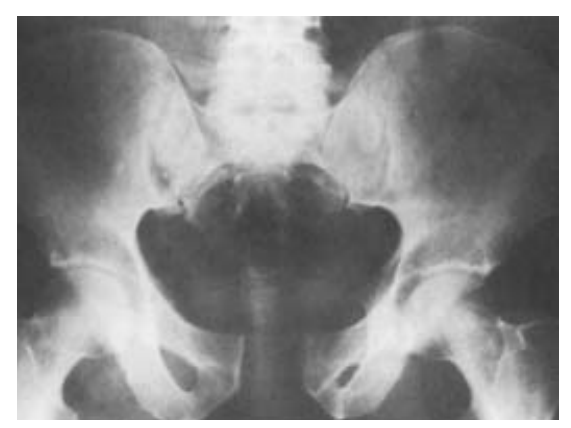

Abb.1c Zustand nach Entfernung des Fixateur externe nach 8 Wochen. Verbleibende Diastase der Symphyse.

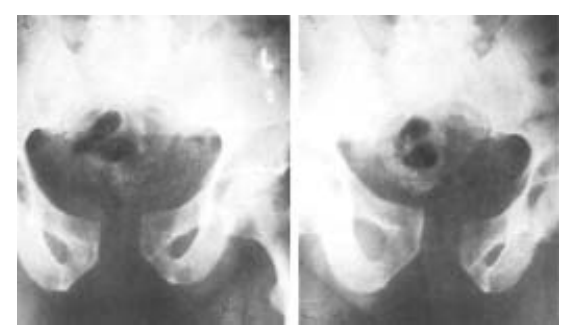

Abb.1d Röntgenaufnahmen im wechselseitigen Einbeinstand, acht Monate nach Unfall. Die Instabilität am vorderen Beckenring ist auf den Aufnahmen gut zu erkennen.
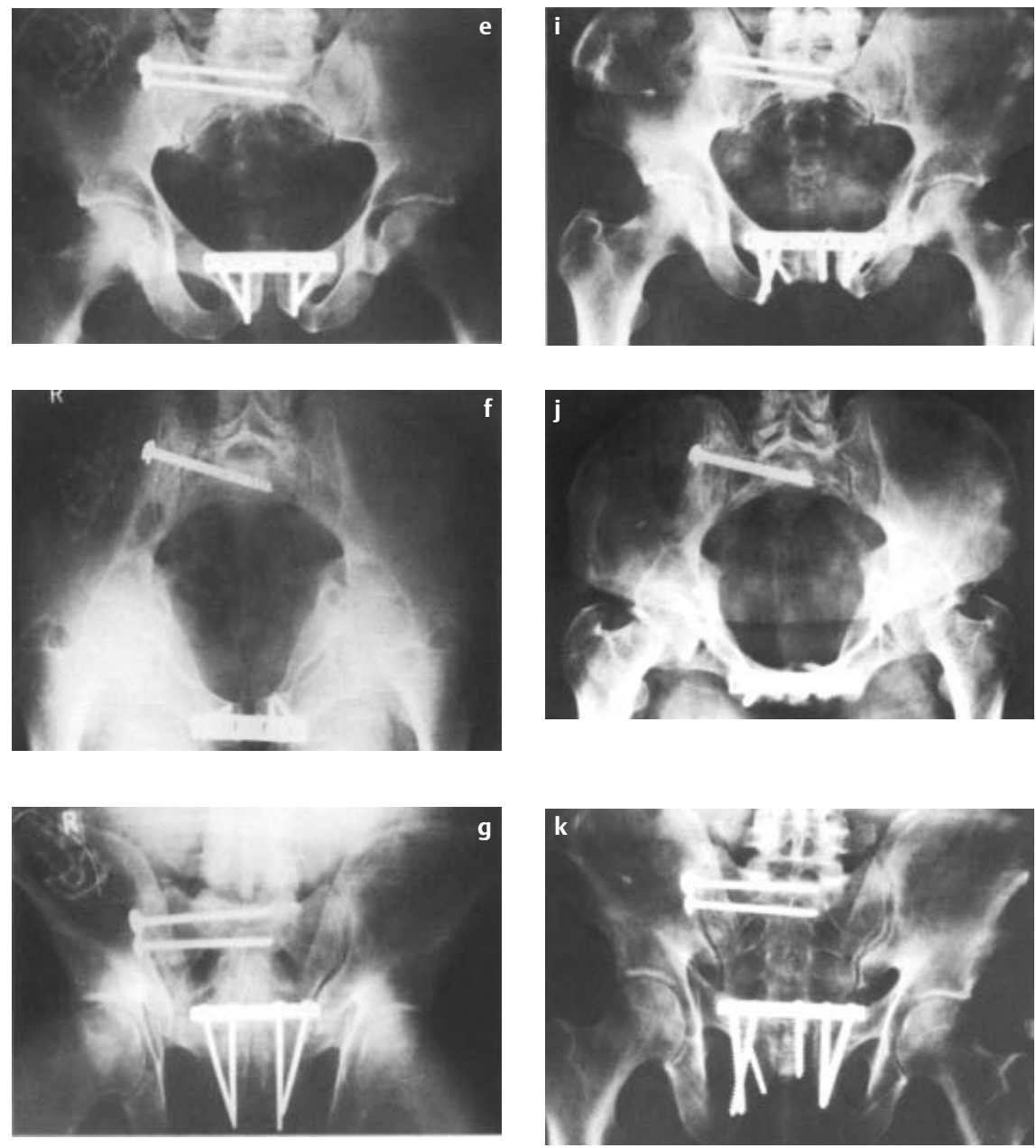

Abb.1e-g Postoperative Aufnahmen nach Stellungskorrektur. Ausräumung des rechten Sakroiliakalgelenkes von ventral. Sakroiliakale Schraubenosteosynthese. Symphysiodese mit Beckenkammspan und Großfragmentplattenosteosynthese. Im weiteren Verlauf Infektrevision der rechten Beckenschaufel mit Ausräumen einer Abszesshöhle an der ehemaligen Verankerungsstelle der Schanz-Schrauben.

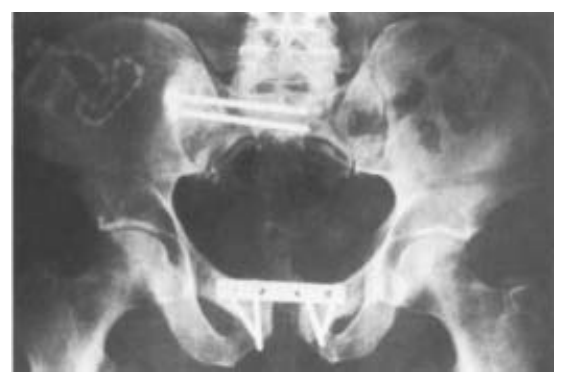

Abb.1h Elf Monate später Implantatversagen der ventralen Platte.

Abb.1i-k Reosteosynthese mit erneuter Beckenkammspaninterposition. 1 Jahr später ist die Symphysiodese nahezu vollständig durchbaut.

kräfte oder Scherkräfte auf die Symphyse, empfiehlt sich die Doppelplattenosteosynthese mittels zwei rechtwinklig zueinanderstehenden Platten, um das Risiko eines Implantatversagens zu reduzieren. Bei chronischen open-Book-Verletzungen mit weit klaffender Symphyse ist es u.U. sinnvoll vor der Osteosynthese des vorderen Beckenrings zunächst über einen retroperitonealen Zugang an der Innenseite der Beckenschaufel Narbengewebe aus dem klaffenden Iliosakralgelenk auszuräumen, um den Verschluss des ventralen Beckenrings anschließend $\mathrm{zu}$ vereinfachen. Wird eine definitive Symphysiodese angestrebt, wird vor der Osteosynthese ein trikortikaler Knochenspan aus der Beckenschaufel in die Symphyse interponiert.

In ähnlicher Technik wird auch bei der transpubischen Instabilität der Pseudarthrosenherd dargestellt, ausgeräumt 


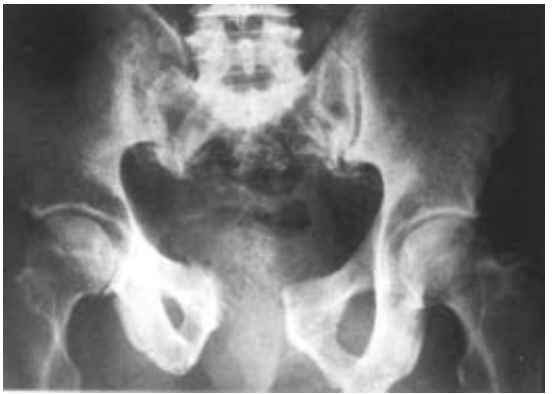

Abb. 2a Symphysensprengung mit vollständiger sakroiliakalen (SI) Luxation rechts bei einem 56-jährigen Patienten. Begleitverletzungen: SHT 3. Grades, Lungenkontusion, Milzruptur und Humerusschaftfraktur links.
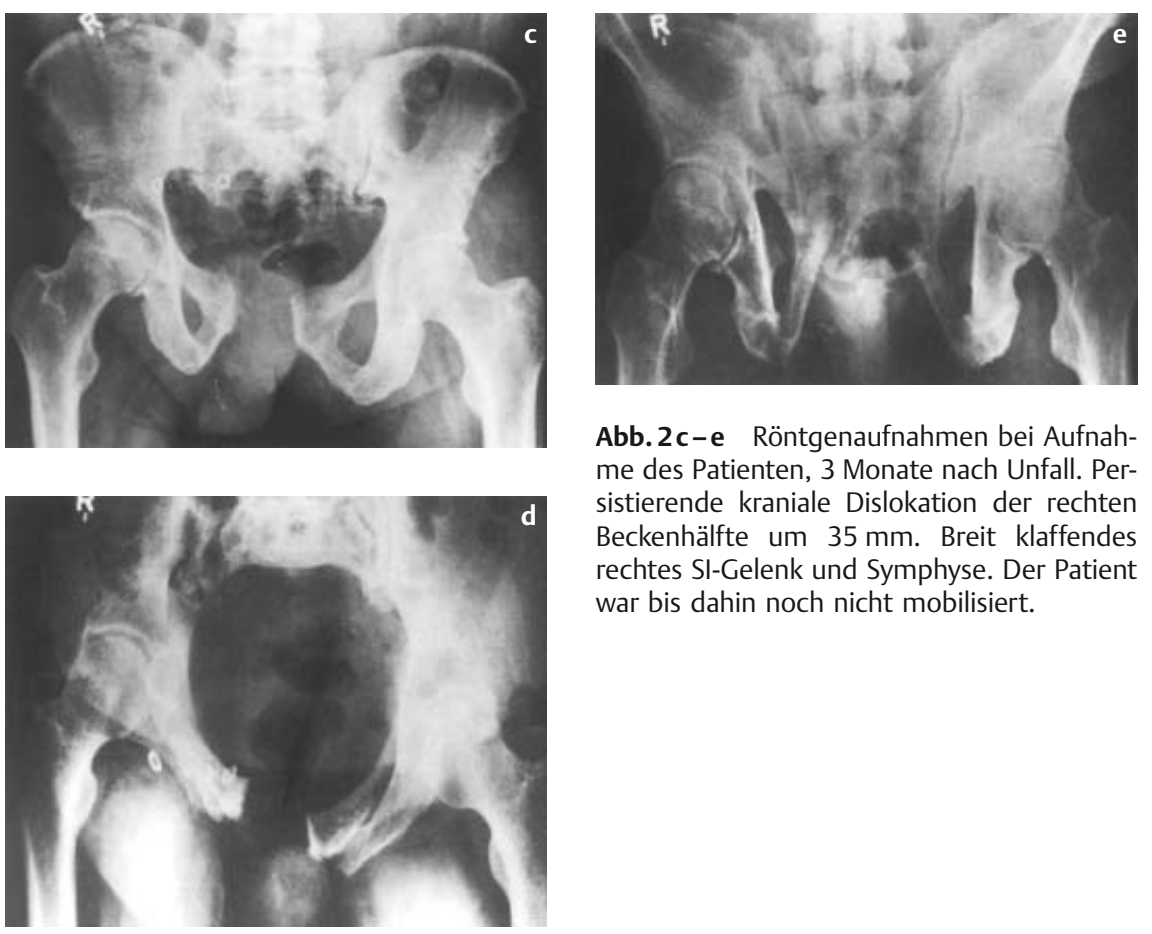

Abb.2c-e Röntgenaufnahmen bei Aufnahme des Patienten, 3 Monate nach Unfall. Persistierende kraniale Dislokation der rechten Beckenhälfte um $35 \mathrm{~mm}$. Breit klaffendes rechtes SI-Gelenk und Symphyse. Der Patient war bis dahin noch nicht mobilisiert.

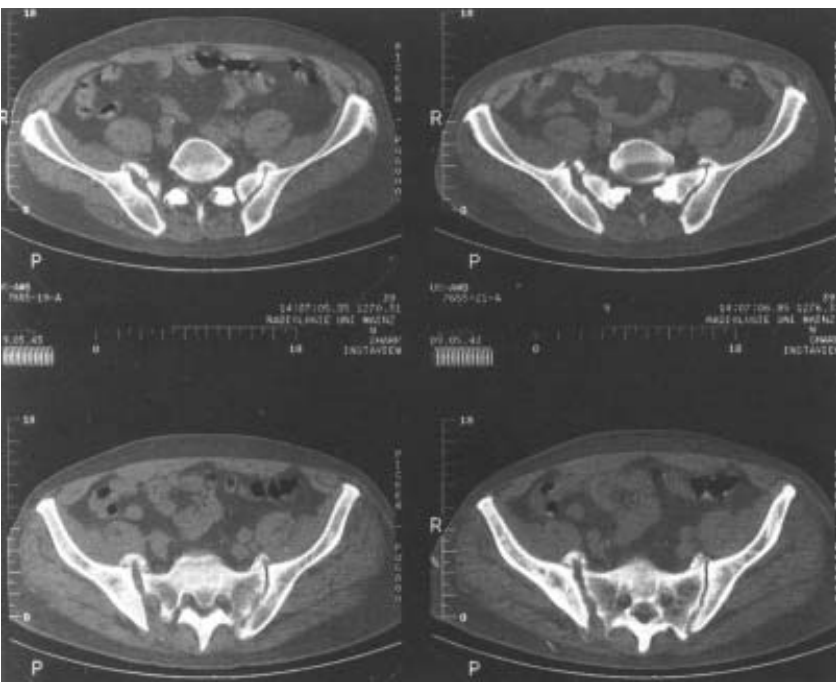

Abb. $2 f$ CT-Aufnahme des hinteren Beckenrings zeigt die noch vorhandene Dislokation des rechten SI-Gelenkes. Deutliche Kallusbildung ventral. Auch links Kallusbildung sichtbar als Zeichen der stattgehabten open-Book-Verletzung.
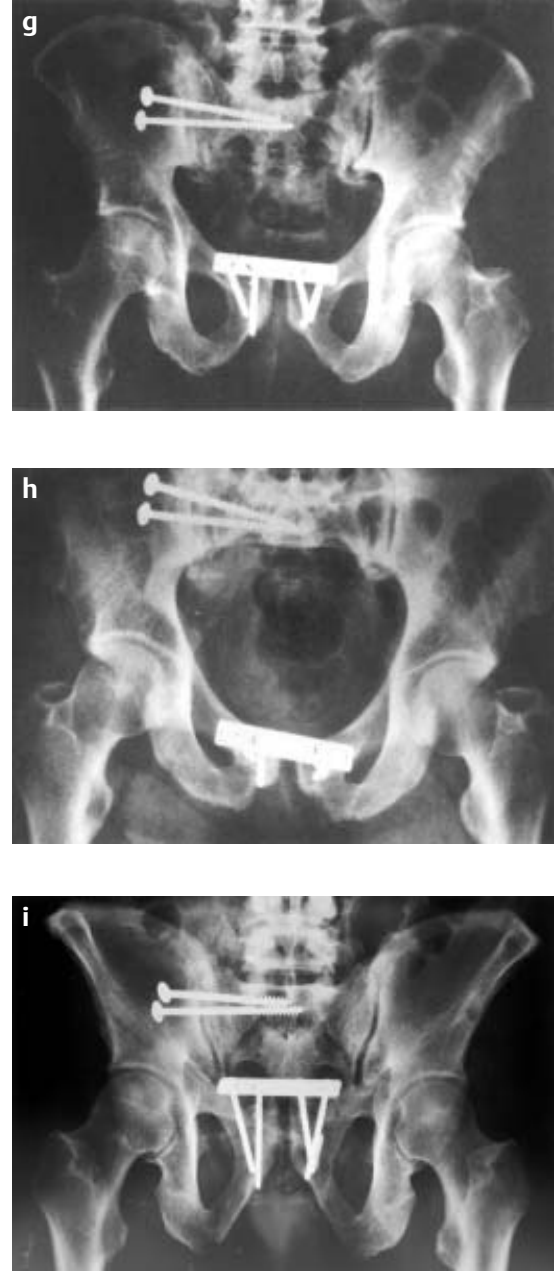

Abb. $\mathbf{2 g - i}$ Zustand 6 Wochen nach Pannusausräumung und Osteosynthese am rechten SI-Gelenk über einen dorsalen Zugang und an der Symphyse über einen Pfannenstielschnitt. Mobilisation des Patienten unter Entlastung des rechten Beines.

und der knöcherne Defekt mit Spongiosa aufgefüllt. Die ossäre Stabilisierung erfolgt mittels Plattenosteosynthese oder besser mit einer langen Zugschraube, welche vom Tuberculum pubicum ausgehend frakturüberquerend im periacetabulären Os ilium verankert wird.

Am dorsalen Beckenring ist je nach Lokalisation der Pseudarthrose ein ventraler oder dorsaler Zugang zu bevorzugen. Instabilitäten des hinteren Beckenrings sind entweder im Sakrum, im Iliosakralgelenk oder im dorsalen Os ilium lokalisiert.

Sakruminstabilitäten werden über einen dorsalen Zugang angegangen. Nach Ausräumen des Pseudarthrosenherdes wird die ehemalige Fraktur durch eine Kom- 
pressionsosteosynthese, gof. in Kombination mit einer Spongiosaplastik, stabilisiert. Die ossäre Stabilisierung erfolgt entweder durch eine sakroiliakale Verschraubung oder durch eine überbrükkende dorsale Plattenosteosynthese.

Das Sakroiliakalgelenk kann sowohl von ventral als auch von dorsal dargestellt werden. Zunächst wird das Gelenk unter Erhalt des Knorpels ausgeräumt und debridiert. Ähnlich wie bei den frischen Verletzungen erfolgt die osteoligamentäre Stabilisierung des Gelenkes bei anteriorem Zugang mittels zwei überbrückenden schmalen Großfragment-DC-Platten. In der Regel reichen kurze 2- bis 3-LochPlatten aus. Wird ein dorsaler Zugang gewählt, kommt die sakroiliakale Schraubenosteosynthese zur Anwendung (Abb. 2).

Dorsale Iliuminstabilitäten werden über einen retroperitonealen Zugang entlang der Innenseite der Beckenschaufel angegangen. Nach Ausräumen von Pannusgewebe aus dem Frakturspalt wird die Fraktur mit Zirkelzangen und/oder Faraboeufklemmen reponiert und anschließend osteosynthetisch fixiert. Die Stabilisierung erfolgt durch eine Plattenosteosynthese, ggf. in Kombination mit einzelnen Zugschrauben, welche frakturüberquerend vom Beckenkamm ausgehend in Richtung der Linea terminalis gebohrt werden. Die Platten werden an der Crista iliaca und der Linea terminalis platziert.

\section{Korrektureingriffe bei Fehlheilungen des Beckens}

Die Rekonstruktion eines fehlverheilten Beckenrings stellt einen außerordentlich schwierigen und anspruchsvollen Eingriff dar. Die Häufigkeit von relevanten Fehlstellungen des Beckenrings liegt nach Literaturangaben bei ca. $5 \%$ [16]. In der Mehrzahl der Fälle handelt es sich um Fehlstellungen nach vertikal instabilen Frakturen, welche primär konservativ behandelt wurden unter zu geringer Zugkraft oder um Frakturen welche nicht ausreichend chirurgisch stabilisiert wurden oder nach unzureichender Reposition osteosynthetisch fixiert worden sind $[2,4,10,13]$.

Die typische Fehlstellung kennzeichnet sich durch einen kraniodorsalen Versatz des verletzten Hemipelvis, wobei die betroffene Beckenhälfte zusätzlich flektiert und innenrotiert ist. Das Hüftgelenk ist demzufolge nach kranial, medial und vorne versetzt (Abb. $\mathbf{3}$ ).
Während isolierte Außen- oder Innenrotationsfehlstellungen in der Regel relativ unproblematisch zu korrigieren sind, ist für die Korrektur komplexerer Fehlstellungen eine präzise präoperative Planung sowie eine aufwendige operationstechnische Logistik erforderlich. Für eine solche Operation müssen ein Operationsteam und ein Operationssaal für einen kompletten Tag reserviert werden. Der Einsatz eines Cell-savers ist bei derartigen Eingriffen anzustreben, das intra-operative Monitoring der peripheren Nervenfunktionen mittels evokierten Potentialen und/oder EMG scheint sinnvoll um Dehnungsschäden, welche durch Distraktion osteoligamentärer Strukturen auftreten können, frühzeitig erkennen, bzw. vermeiden können [5]. Allerdings ist diese Methode nach bisherigen Erfahrungen noch nicht als sehr zuverlässig anzusehen [8].

Korrekturen komplexer Fehlheilungen des Beckens erfolgen immer in mehreren Schritten. Zunächst müssen die Fehlheilungen sowohl im vorderen als auch im hinteren Beckenring gelöst werden, bevor mit der Stellungskorrektur und der Osteosynthese begonnen werden kann. Die Entscheidung, ob die Osteotomie zunächst ventral und dann dorsal vorgenommen wird oder umgekehrt, ist abhängig von der Art der Fehlstellung und von der geplanten Vorgehensweise.

Bedingt durch das schrittweise Vorgehen, muss der Patient intraoperativ von Bauchlage in Rückenlage gebracht werden und umgekehrt, wobei die einzelnen Zugänge vor dem Umlagern provisorisch verschlossen und später wieder neu eröffnet werden müssen.

Die ventrale transpubische Osteotomie erfolgt uni- oder bilateral, Kallus- und Narbengewebe müssen abgetragen werden um die fehlverheilte Beckenhälfte zu lösen und kontrakte Ligamente müssen durchtrennt werden. Bedingt durch die Verzerrung der normalen Anatomie befinden sich die neurovaskulären Strukturen häufig in atypischer Lage. Da diese Strukturen zudem oftmals durch sehr derbes Narben- oder Kallusgewebe umgeben sind, muss sehr umsichtig vorgegangen werden, um während der Präparation Nerv- oder Gefäßverletzungen zu vermeiden.

Neurologische Probleme sind die Hauptursachen postoperativer Beschwerden. Nervenschäden können entweder durch die chirurgische Präparation, durch Traktion während der Reposition oder wäh- rend der Osteosynthese auftreten. Deshalb empfiehlt es sich, die betroffenen Beckenhälfte, nachdem diese ausreichend mobilisiert worden ist, nur sehr langsam und umsichtig in ihre ursprüngliche anatomische Stellung zurückzubringen, um es den umliegenden Weichteilstrukturen zu ermöglichen, sich zu dehnen und der geänderten Anatomie anzupassen. Für die Mobilisation und Stellungskorrektur sind der Fixateur externe und der große Distraktor von großer Hilfe. Die Reposition sollte immer unter Bildwandlerkontrolle in verschiedenen Ebenen erfolgen. Intraoperative Überprüfung der Nervenfunktionen mit evokierten Potentialen und/oder EMG kann frühzeitig auf drohende neurologische Ausfälle hinweisen. Auch sollte intraoperativ das gleichseitige Bein in einer Beugestellung des Kniegelenkes gelagert werden um die entsprechenden neurovaskulären Strukturen zu entspannen.

Die einzelnen Schritte der Osteosynthese müssen mit dem Bildwandler kontrolliert werden um Fehlbohrungen oder Fehllagen von Implantaten zu vermeiden.

Da nach der Korrektur teilweise erhebliche Kräfte auf die Osteosynthese einwirken, die Implantate sich in dem oftmals osteoporotischen Knochen nicht immer optimal verankern lassen und zudem mit längeren Heilungszeiten zu rechnen ist, sollte eine sichere und $u$. U. aufwendigere Fixierung als bei frischen Verletzungen vorgenommen werden. Passive Mobilisationsübungen werden umsichtig unter Entlastung des Gelenkes vorgenommen. Beim Gehen und Stehen sollte eine Abrollbelastung der operierten Beckenhälfte für mindestens drei Monate beibehalten werden.

Dennoch ist die Implantatlockerung mit nachfolgender Pseudarthrosenbildung eine wesentliche Komplikation nach Korrekturosteosynthesen am Becken. Kommt es zu einer Auslockerung der Implantate oder einem Implantatversagen ventral- oder dorsalseitig, muss in der Regel stets auch eine Lockerung der gegenseitigen Osteosynthese angenommen werden. Eine erneute Revision sollte somit dann an beiden Korrekturstellen erfolgen.

Postoperative Wundprobleme, Hämatombildungen oder Infektionen können in ihrer Häufigkeit reduziert werden durch weichteilschonendes intraoperatives Vorgehen, ausreichender Spülung mit sorgfältigem Debridement des Operationsgebietes und Einlagen von Redon- 


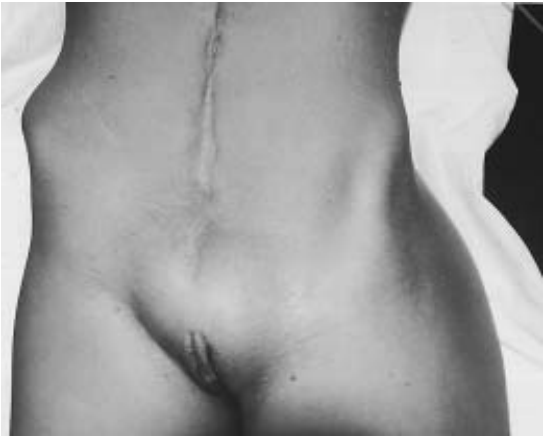

Abb.3a 29jährige Patientin mit fehlverheilter Beckenringfraktur 22 Jahre nach Überrolltrauma - klinischer Befund.

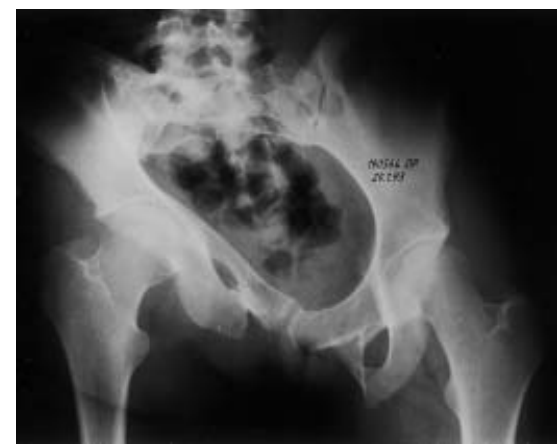

Abb.3b Radiologische Ausgangssituation in der Becken a.p.-Projektion.

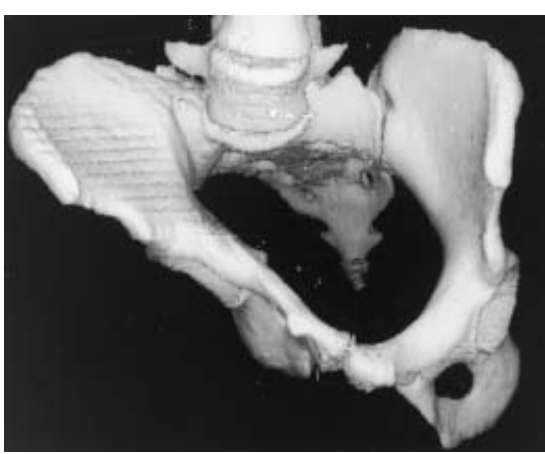

Abb.3c 3D-Rekonstruktion zeigt die in Flexion und Innenrotation fehlverheilte rechte Beckenhälfte, welche zudem ein Vertikalversatz aufweist.
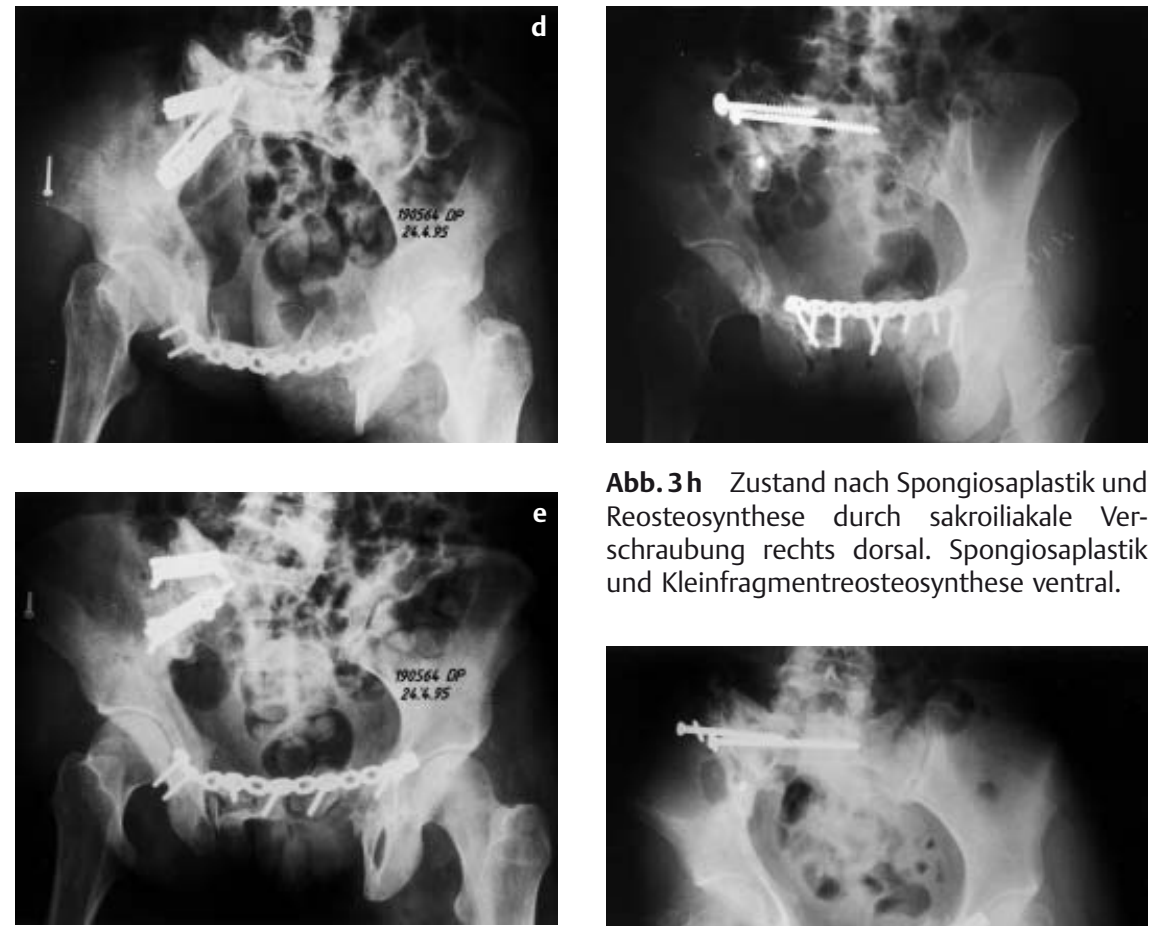

Abb.3d,e Zustand nach Rekonstruktion in der Beckenübersichts und -Ausgangsaufnahme. Beidseitige transpubische Osteotomie mit Kleinfragmentplattenosteosynthese ventral und Doppelplattenosteosynthese der rechts dorsalen Osteotomie.
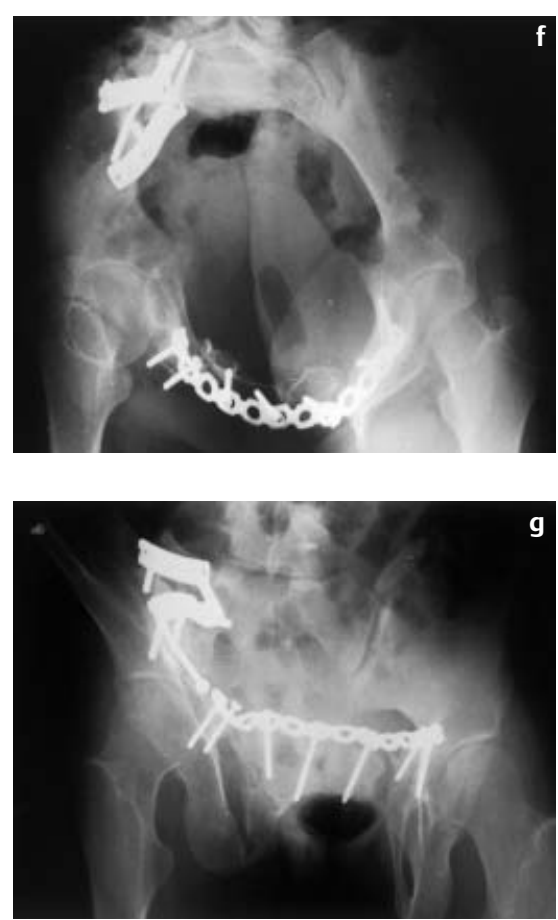

Abb.3f, g Plattenbruch ventral mit Auslockerung der ventralen und dorsalen Schrauben, 22 Monate nach Korrekturosteotomie. Beckeneingang- und Beckenausgangsaufnahme.
Abb.3h Zustand nach Spongiosaplastik und Reosteosynthese durch sakroiliakale Verschraubung rechts dorsal. Spongiosaplastik und Kleinfragmentreosteosynthese ventral.

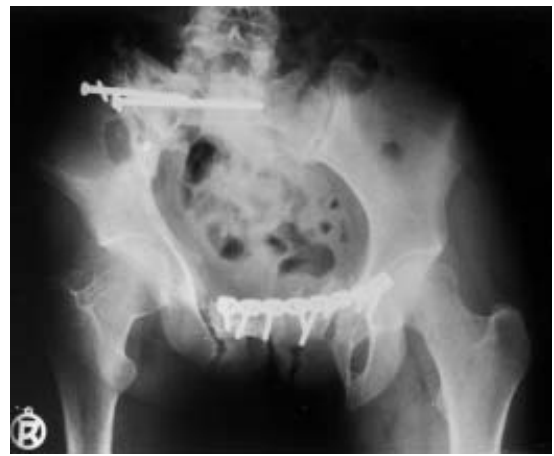

Abb.3i Implantatlockerung am dorsalen Beckenring mit ausbleibender Knochenheilung.

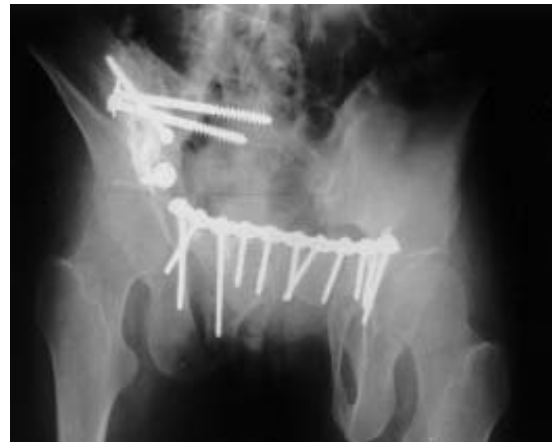

Abb.3j Zustand nach erneuter Reosteosynthese, 33 Monate nach Erstoperation. Neuplazierung der sakroiliakalen Schrauben mit Spongiosaplastik. Spongiosaplastik rechts ventral und Reosteosynthese mit einer Platte, welche jetzt beide ehemalige Osteotomiebereiche überbrückt. Diesmal kommt es zu einer vollständigen knöchernen Konsolidierung der ventralen und dorsalen Osteotomie. 
drainagen in ausreichender Anzahl und Durchmesser in die einzelnen Wundhöhlen.

\section{Fazit}

Chirurgische Korrekturen von Fehlheilungen des Beckenrings sind seltene aber komplexe und anspruchsvolle Eingriffe. Weniger häufig als bei frischen Verletzungen können die normale Anatomie und Funktionalität wiederhergestellt werden. Sowohl dem Chirurgen als auch dem Patienten sollte bewusst sein, dass es sich bei den Korrekturosteosynthesen um aufwendige und komplikationsträchtige Eingriffe handelt. Umfassende Erfahrungen bei der chirurgischen Behandlung akuter Beckenring- und Acetabulumfrakturen sind für das Gelingen dieser Korrekturmaßnahmen am Becken unabdingbare Voraussetzungen.

\section{Literatur}

${ }^{1}$ Cole JD, Blum DA, Ansel LJ. Outcome after fixation of unstable posterior pelvic ring injuries. Clin Orthop 1996; 329: 160-179

${ }^{2}$ Ebraheim NA, Biyani A, Wong F. Nonunion of pelvic fractures. J Trauma 1998; 44: $202-$ 204

${ }^{3}$ Gautier E, Ganz R. Spätrekonstruktive Eingriffe nach Beckenverletzungen mit Fehlheilung. OP-Journal 1993; 9: 46-53

${ }^{4}$ Gautier E, Rommens PM, Matta JM. Late reconstruction acter pelvic ring injuries. Injury 27, Suppl 1996; 2: B39-B46

${ }^{5}$ Helfet DL, Koval, KJ, Hissa EA, Patterson S, Dipasquale T, Sanders R. Intraoperative somatosensory evoked potential monitoring during acute pelvic fracture surgery. J Orthop Trauma 1995; 9: 28-34

${ }^{6}$ Hessmann MH, Mayer A, Rommens PM. Internal fixation of posterior pelvic ring lesions. Osteo Internat 1999; 7: 34-44

${ }^{7}$ Kreitner KF, Rommens PM: radiologische Diagnostik von Becken- und Acetabulumfrakturen. Osteo Internat 1999; 7: 3-12

${ }^{8}$ Matta JM, Dickson KF, Markovich D. Surgical treatment of pelvic nonunions or malunions. Clin Orthop 1996; 329: 129-140
${ }^{9}$ Matta JM, Tornetta P. Internal fixation of unstable pelvic ring injuries. Clin Orthop 1996; 329: $129-140$

${ }^{10}$ Mears DC. Das Management von Beckenpseudarthrosen und Beckenfehlstellungen. Orthopädie 1996; 25: 441 - 449

${ }^{11}$ Moore RS, Stover MD, Matta, JM. Later posterior instability of the pelvis after resection of the symphysis pubis for the treatment of osteitis pubis. A report of two cases. J Bone Joint Surg 1998; 80 A: $1043-1048$

${ }^{12}$ Olson SA, Pollak A.N. Assessment of pelvic ring stability after injury. Clin Orthop 1996; 329: 15-27

${ }^{13}$ Pennal GF, Massiah KA. Nonunion and delayed union of fractures of the pelvis. Clin Orthop 1980; 151: 124-129

${ }^{14}$ Rommens PM. Pelvic ring injuries: a challenge for the trauma surgeon. Acta Chir Belg 1996; 96: 78-84

${ }^{15}$ Rommens PM, Hessmann, MH. Early ersus delayed surgery for muskuloskeletal trauma in polytraumatized patients. Curr Opin Crit Care 1998; 4: 424-428

${ }^{16}$ Tile, M.: Fractures of the pelvis and acetabulum, 2nd Edition. Lippincott, Williams, Wilkins Verlag 1995

\section{Dr. med. M. H. Hessmann Oberarzt der Klinik}

Prof. Dr. med. P. M. Rommens Direktor der Klinik

Klinik und Poliklinik für Unfallchirurgie Johannes-Gutenberg-Universität Langenbeckstraße 1

55101 Mainz 